\title{
INFLUENCE OF TIME OF DAY ON REACTIONS TO CYCLING AT A FIXED HIGH INTENSITY
}

\author{
T. REILLY, BA, DipPE, MSc, PhD, MIBiol. and Claire BAXTER, BSc*
}

Department of Sport and Recreation Studies, Liverpool Polytechnic, Byrom Street Liverpool L3 $3 A F$

\begin{abstract}
The circadian cycle in all-out competitive performance may be due to changes in motivational drive to tolerate strenuous exercise rather than to rhythms in maximal physiological functions. This experiment explored the hypothesis that a fixed relative loading of high intensity aerobic effort could be sustained for longer in the evening compared with the early morning. Eight females cycled to exhaustion at $95 \% \mathrm{VO}_{2} \max$ at $06.30 \mathrm{~h}$. and at $22.00 \mathrm{~h}$. after a $5 \mathrm{~min}$. moderate load of $40 \% \mathrm{VO}_{2}$ max. Oral temperature was $0.4^{\circ} \mathrm{C}$ higher at $22.00 \mathrm{~h}$. compared with the earlier time. Perceived exertion showed no significant effect of time of day at either work load but exercise tolerance time, total work done and peak lactate production were significantly greater at $22.00 \mathrm{~h}$. $(p=0.05)$. Perceived exertion at the high load was significantly related to endurance time $(r=-0.77)$ and to the rating at the low work level $(r=0.75)$. Peak lactate was not significantly correlated with the time to exhaustion $(p>0.05)$. It was concluded that superior exercise performance in the evening may be attributed to a greater tolerance for high intensity exercise which is closely associated with the acrophase in body temperature.
\end{abstract}

\section{INTRODUCTION}

As shown in our paper on swimming (Reilly and Baxter, 1983) all-out exercise performance tends to exhibit a circadian rhythm closely in phase with that of body temperature. The fluctuations in performance do not seem to be accompanied by changes in aerobic power or muscular efficiency (Reilly and Brooks, 1982). As maximal aerobic power output cannot be sustained for long, the circadian rhythm in performance may be because of a willingness to withstand set near-maximal loadings for longer at the optimal time of day for exercise. In that case the effect of the time of day on exercise performance may have a more immediate motivational rather than metabolic explanation and this provided the rationale for the present study.

It was hypothesised that subjects are reluctant to exercise at a given strenuous level for as long at the time of day when body temperature is low compared with the performance time when body temperature is near the peak of its circadian curve. A more acute subjective reaction to exercise at the former time was predicted.

\section{METHODS}

Eight females aged 18-21 (mean age 19.5) volunteered to undertake an experimental exercise test of high intensity to exhaustion on two separate occasions, one at $06.30 \mathrm{~h}$. and the other at $22.00 \mathrm{~h}$. These times were

\footnotetext{
"Present address:

University Dept. of Orthopaedic and Accident Surgery, Royal Liverpool Hospital,

Liverpool L7 8XP
}

chosen in the light of results in an earlier investigation (Baxter and Reilly, 1983). The experiment necessitated the prior measurement of $\mathrm{VO}_{2}$ max which was done in the two weeks before the first test. An automated gas analysis system (P. K. Morgan Ltd.), calibrated prior to each maximal test, was used. The protocol for assessment of $\mathrm{VO}_{2}$ max involved an incremental work test to exhaustion on a Lode electrically braked cycle ergometer: Åstrand and Rodahl's (1977) levelling off criterion was used as evidence that $\mathrm{VO}_{2} \max$ was attained. The work loads corresponding to $40 \%$ and $95 \%$ of the $\mathrm{VO}_{2}$ max were calculated by linear regression analysis of the work rate/ $\mathrm{VO}_{2}$ relationship during this exercise test.

The experimental test protocol involved a preliminary $5 \mathrm{~min}$. period on the Lode cycle ergometer at a work rate previously established to represent a $\mathrm{VO}_{2} 40 \%$ of maximum. This intensity was chosen to represent light exercise below the anaerobic threshold as defined by Wasserman et al (1973). Perceived exertion was rated according to Borg (1970) in the fourth minute of this work load. Subjects continued cycling as the exercise intensity was elevated to $95 \%$ of $\mathrm{VO}_{2}$ max in ramp fashion at a rate of $3.66 \% \mathrm{VO}_{2} \operatorname{max~s} \mathrm{s}^{-1}$. This severity was selected in anticipation that it could be sustained for a duration approximately similar to a $400 \mathrm{~m}$. swim time. Time to exhaustion on the $95 \% \mathrm{VO}_{2}$ max work load was determined at the point the subject was unable to continue pedalling: perceived exertion was again rated near this point. Total work performed was calculated from the power output which was pre-set, and the time from commencing exercise to reaching exhaustion.

Oral temperature was determined pre-exercise by means of a mercury thermometer placed sub-lingually 
for $3 \mathrm{~min}$. Two $20 \mu \mathrm{l}$ finger tip capillary blood samples were obtained $3 \mathrm{~min}$. post-exercise for lactate determination by an enzymatic method (Bergmeyer, 1974). The laboratory temperature was constant at $21.5^{\circ} \mathrm{C}$ (S.D. $=0.2^{\circ} \mathrm{C}$ ) for all tests.

Means and standard deviations were calculated for morning and evening values. A time of day effect was investigated using both a two-way analysis of variance and a non-parametric sign test. $P$ values of 0.05 were taken as indicating statistical significance.

\section{RESULTS}

Body weight and maximal physiological measures are presented for all subjects in Table I. Mean $\mathrm{VO}_{2} \max$ of subjects was 41 ( \pm S.D. $=9$ ) $\mathrm{ml} \cdot \mathrm{kg}^{-1} \mathrm{~min}^{-1}$ or 2.41 $( \pm$ S.D. $=0.34) \mathrm{I} \cdot \mathrm{min}^{-1}$. Mean VE $\max$ was $105( \pm$ S.D. = 17.5) I. $\mathrm{min}^{-1}:$ mean maximal heart rate was 185 ( \pm S.D. $=6$ ) beats $\min ^{-1}$. Descriptive data and the results of the analysis of variance are presented in Table II. Significant time of day effects were found for pre-start oral temperature and peak lactate production, being $0.4^{\circ} \mathrm{C}$ and 1.7 $\mathrm{mM}$ higher in the evening respectively. The variance among subjects was non-significant for all variables. Mean time to exhaustion was $68 \%$ longer in the evening though the result was significant only when non-parametric analysis was performed $(p=0.05)$. Similarly, the total work done was $41 \%$ greater in the evening, a result significant only for the sign test $(p=0.05)$.
The single subject capable of exercising for longer in the morning was also the one exception producing higher lactate levels at that time.

Time to exhaustion was not significantly correlated with peak lactate $(r=0.14)$. A significant correlation $(r=-0.77)$ was found between the rating of perceived exertion at $95 \% \mathrm{VO}_{2}$ max and endurance time. A correlation coefficient of $\mathbf{0 . 7 5}$ was obtained for ratings of perceived exertion at the $40 \%$ and $95 \% \mathrm{VO}_{2} \max$ work loads, but mean values did not vary significantly between times of day for either of these work intensities.

\section{DISCUSSION}

The experimental findings offer some support for the concept of a motivational component affecting strenuous exercise performance differentially according to the time of day. Contrary to expectations the perceived exertion did not seem to be a limiting factor as it was constant with time of day at each of the work loads studied. Recent reports of a circadian rhythm in the subjective reaction to exercise included ratings associated with a given heart rate when power output may not have been constant (Faria and Drummond, 1982); and ratings during bouts of light work repeated every 4 h. (Reilly and Young, 1982), a paradigm which may have involved a cumulative fatigue effect. The longer performance in the evening with equivalent ratings of

\section{TABLE I}

Body weight and maximal physiological measure of subjects.

$\begin{array}{lcccc}\text { Subject } & \text { Subject weight }(\mathrm{kg}) & \mathrm{VO}_{2} \max \left(1 . \mathrm{min}^{-1}\right) \text { STPD } & V E \max \left(1 . \mathrm{min}^{-1}\right) \text { BTPS } & \text { fH } \max \left(b^{2} \operatorname{mits}^{-1}\right) \\ \text { BC } & 53.7 & 2.89 & 91.6 & 188 \\ \text { JH } & 53.2 & 2.74 & 101.3 & 180 \\ \text { LN } & 60.0 & 2.12 & 124.8 & 186 \\ \text { KF } & 66.0 & 2.22 & 113.2 & 186 \\ \text { DS } & 77.0 & 2.64 & 121.8 & 198 \\ \text { LJ } & 54.0 & 2.52 & 70.7 & 182 \\ \text { KB } & 63.0 & 1.90 & 106.1 & 180 \\ \text { LH } & 53.2 & 2.26 & 110.2 & 180\end{array}$

\section{TABLE II}

Descriptive data (mean \pm SD) for dependent variables in the morning and evening $(n=8)$ and summary of ANOVA results.

\section{Variable}

Oral temperature $\left({ }^{\circ} \mathrm{C}\right)$

Time to exhaustion (s)

Perceived exertion at $40 \% \mathrm{VO}_{2} \max$ Perceived exertion at $95 \% \mathrm{VO}_{2} \max$ Peak Lactate (mM)

Total work done (kpm)
$06.30 h$

$\begin{array}{rlr}36.2 & \pm & 0.5 \\ 259.6 & \pm & 150.2 \\ 10.6 & \pm & 1.6 \\ 17.4 & \pm & 1.1 \\ 8.15 & \pm & 1.48 \\ 5277 & \pm 1539\end{array}$

$22.00 \mathrm{~h}$.

$\begin{array}{rlr}36.6 & \pm & 0.3 \\ 435.6 & \pm & 432.0 \\ 10.1 & \pm & 1.0 \\ 16.6 & \pm & 1.6 \\ 9.83 & \pm & 1.18 \\ 7424 & \pm 4229\end{array}$

ANOVA Results
Time of Day $\quad$ Subjects

$p<0.05$

NS

NS

NS

$p<0.05$

NS 
exertion fits a drive theory interpretation of findings rather than an arousal model (Martens, 1974), subjects being better prepared to tolerate a fixed level of subjective discomfort at that time.

Individual tolerance to blood lactate levels cannot account for the variation in performance time between the morning and evening since no significant relationship was observed between these two variables. The higher lactate levels in the evening were probably attributable to the greater amount of work performed, though the existence of a circadian rhythm in anaerobic capacity cannot be excluded. The significant negative correlation between perceived exertion and endurance time was because the more capable individuals sustained the exercise test at a lower rating of exertion than their less fit counterparts who showed greater subjective distress at exhaustion and desisted earlier.

The findings have to be treated tentatively because of the size of the sample used and the confinement of significant results to the non-parametric test. They suggest that a fixed intensity of hard exercise may be tolerated for longer at the time of day when body temperature is high. A possible implication is that vigorous training sessions may be more effective when conducted in the evening rather than early morning, because more work may be performed at that time before voluntary exhaustion occurs. The question of the causal mechanisms involved and the question of transfer of the greater exercise tolerance time to training conditions remain to be addressed.

\section{REFERENCES}

Åstrand, P. O. and Rodahl, K., 1977. Textbook of Work Physiology. (New York: McGraw Hill).

Baxter, C. and Reilly, T., 1983 "Influence of time of day on all-out swimming". Brit.J.Sports Med. 17:

Bergmeyer, H. U., 1974. Methods of Enzymatic Analysis. (New York: Academic Press).

Borg, G., 1970 "Perceived exertion as an indicator of somatic stress". Scandinavian Journal of Rehabilitation Medicine 2: $92-98$.

Faria, I. E. and Drummond, B. J., 1982 "Circadian change in resting heart rate and body temperature, maximal oxygen consumption and perceived exertion". Ergonomics 25: 381-386.

Martens, R., 1974 "Arousal and motor performance". In: Exercise and Sport Sciences Reviews. Ed. J. Wilmore. (New York: Academic Press).

Reilly, T. and Brooks, G. A., 1982 "Investigation of circadian rhythms in metabolic responses to exercise". Ergonomics 19: $1093-1107$.

Reilly, T. and Young, K., 1982 "Digit summation, perceived exertion, and time of day under submaximal exercise conditions". In: Proceedings 20th International Congress of Applied Psychology. Abstracts (Edinburgh) pp. 344.

Wasserman, K., Whipp, B. J., Koyal, S. N. and Beaver, W. L., 1973 "Anaerobic threshold and respiratory gas exchange during exercise". J.Appl.Physiol. 35: 236-243. 